\title{
Imaging Ultrafast Demagnetization Dynamics after a Spatially Localized Optical Excitation
}

\author{
C. von Korff Schmising, ${ }^{1, *}$ B. Pfau, ${ }^{1, \dagger}$ M. Schneider, ${ }^{1}$ C. M. Günther, ${ }^{1}$ M. Giovannella, ${ }^{1,2}$ J. Perron, ${ }^{3,4}$ B. Vodungbo, ${ }^{3,4}$ \\ L. Müller, ${ }^{5}$ F. Capotondi ${ }^{6}$ E. Pedersoli, ${ }^{6}$ N. Mahne, ${ }^{6}$ J. Lüning, ${ }^{3,4}$ and S. Eisebitt ${ }^{1,7}$ \\ ${ }^{1}$ Institut für Optik und Atomare Physik, Technische Universität Berlin, 10623 Berlin, Germany \\ ${ }^{2}$ Dipartimento di Fisica, Università di Pisa, Largo Bruno Pontecorvo 3, 56127 Pisa, Italy \\ ${ }^{3}$ Sorbonne Universités, UPMC Université Paris 06, UMR 7614, LCPMR, 75005 Paris, France \\ ${ }^{4}$ CNRS, UMR 7614, LCPMR, 75005 Paris, France \\ ${ }^{5}$ Deutsches Elektronen-Synchrotron DESY, 22607 Hamburg, Germany \\ ${ }^{6}$ Elettra-Sincrotrone Trieste, Strada Statale 14-km 163.5, 34149 Basovizza, Trieste, Italy \\ ${ }^{7}$ Helmholtz-Zentrum Berlin für Materialien und Energie, 14109 Berlin, Germany \\ ${ }^{8}$ Division of Synchrotron Radiation Research, Department of Physics, Lund University, S-22100 Lund, Sweden
}

(Received 24 October 2013; published 29 May 2014)

\begin{abstract}
Ultrashort, coherent x-ray pulses of a free-electron laser are used to holographically image the magnetization dynamics within a magnetic domain pattern after creation of a localized excitation via an optical standing wave. We observe a spatially confined reduction of the magnetization within a couple of hundred femtoseconds followed by its slower recovery. Additionally, the experimental results show evidence of a spatial evolution of magnetization, which we attribute to ultrafast transport of nonequilibrium spin-polarized electrons for early times and to a fluence-dependent remagnetization rate for later times.
\end{abstract}

DOI: $10.1103 /$ PhysRevLett.112.217203

PACS numbers: 75.78.Jp, 42.40.Kw, 75.25.-j, 78.70.Ck

Progress in the field of light-induced, ultrafast manipulation of magnetic order has recently led to all-optical, ultrafast magnetic switching [1-3] and to an increased control of its dynamics by designing tailored nanostructured samples [4-7] as well as by exploiting nanoscale magnetic inhomogeneities $[8,9]$. The influence of interfaces between different materials and magnetic domain boundaries has cast doubt on our theoretical understanding of the underlying fundamental mechanism responsible for femtosecond magnetization dynamics. The model explaining the ultrafast loss of magnetic order after optical excitation by (e.g., electron-phonon or impurity-mediated) spin-flip scattering events [10] has in part been challenged by an approach based on nonlocal superdiffusive spin transport [11]. In spite of their very different microscopic origins, both have been successful in explaining a wide range of experimental data, suggesting that both mechanisms play an important role and that their respective magnitudes depend on the specific experimental conditions [7]. More specifically, in the case of superdiffusive spin transport, energy- and spin-dependent electron lifetimes and velocities induce spin-polarized currents, leading to significant ultrafast spatial rearrangement of magnetic order.

To gain control of magnetization dynamics and all-optical switching in the lateral dimension, one relies on nanometer localization of the optical excitation, as well as detailed knowledge on how (spin-polarized) electron currents lead to a spatial transfer of magnetization. Technologically this plays an important role not only for all-optical approaches, but also for heat-assisted magnetic recording, which has the potential to increase the magnetic recording density by lowering the coercitivity of high-anisotropy materials [12]. Necessary to this end, it is required to deliver the optical energy to a sub100-nm spot size, i.e., far beyond the diffraction limit of optical light. The most successful approaches include localization of the evanescent light from near-field optical probes [13], using metallic plates to excite surface plasmons [14,15] or a combination thereof [16].

Here, we implement time-resolved Fourier transform holography (FTH) [17] and exploit x-ray magnetic circular dichroism (XMCD) to directly image the magnetization dynamics induced by a deliberately spatially localized excitation via an optical standing wave. In addition to a spatially confined reduction of the magnetization and its subsequent slower recovery, evidence is found for concurrent changes in the spatial magnetization profile, suggesting the presence of ultrafast lateral transport of spin-polarized electrons on a nanometer length scale. This novel experimental approach allows studying magnetization dynamics directly with tailored magnetic inhomogeneities in two dimensions.

The experiment was performed at the DIPROI beam line at the free-electron laser (FEL) facility FERMI in Trieste, Italy [18]. Circularly polarized, coherent, 100 -fs short, soft X-ray pulses tuned to the $M$ edge of Cobalt at $20.8 \mathrm{~nm}$ $(58.9 \mathrm{eV})$ were used to image the magnetic domain pattern via FTH [17]. The average intensity of the $\mathrm{x}$-ray pulses incident on the sample surface was reduced by a gas absorber and aluminum filters to $<0.1 \mu \mathrm{J}$ (equivalent to $<1 \times 10^{10}$ photons) to minimize damage and $\mathrm{x}$-ray-induced changes of 
the magnetic state [19]. The focal size of the FEL pulses was approximately $50 \mu \mathrm{m}$ (FWHM). $P$-polarized, 100-fs short optical pulses centered at a wavelength of $\lambda=780 \mathrm{~nm}$ with an energy of $0.2 \mu \mathrm{J}$ were incident on the sample under an angle of $45^{\circ}$. The laser footprint on the sample of $160 \times$ $140 \mu \mathrm{m}^{2}$ (FWHM) resulted in a low excitation fluence of approximately $1.3 \mathrm{~mJ} \mathrm{~cm}^{-2}$. The pump pulses were derived from the FEL seed laser and, hence, were inherently time synchronized to the $\mathrm{x}$-ray probe with a $10-\mathrm{Hz}$ repetition rate. The temporal overlap between the $\mathrm{x}$-ray pulse and the IR laser was determined by a cross-correlation measurement of the optical reflectivity drop of GaAs induced by the FEL radiation [20]. Because of the noncollinearity of the pump and probe light, the uncertainty of time delay zero due to this measurement amounts to $\approx \pm 250 \mathrm{fs}$. However, it is important to note that the overall temporal resolution of our experiment is only limited by the pulse duration of the pump and probe pulses, i.e., on the order of 100 fs. The FTH sample structure was homogeneously illuminated by the FEL pulses, and the hologram or interference pattern generated by the object and reference waves was recorded by a $27.6 \times 27.6 \mathrm{~mm}^{2}(2048 \times 2048$ pixels $)$ large in-vacuum, back-illuminated charged coupled device (CCD) camera placed $50 \mathrm{~mm}$ behind the sample. A beam stop mounted in front of the CCD camera blocked the intense direct beam. Two holograms with opposite helicities of the incoming $\mathrm{x}$ rays were recorded for each time delay. Each hologram is an accumulation of 3500 pulses. The holographic mask of the sample differs in so far from standard implementations [17] that (i) the field of view has an elliptical shape $\left(1.2 \times 2.4 \mu \mathrm{m}^{2}\right)$ and (ii) two gold nanoislands with dimensions $300 \times 300 \times 100 \mathrm{~nm}^{3}$ separated by a 70 -nm-wide gap are left inside the object hole. A magnetic $\mathrm{Co} / \mathrm{Pd}$ multilayer $\left[\operatorname{Pd}(20) /[\mathrm{Co}(4) / \operatorname{Pd}(2)]_{20} / \operatorname{Al}(30)(\AA)\right]$ showing out-of-plane magnetic anisotropy was deposited on the backside of the $\mathrm{Si}_{3} \mathrm{~N}_{4}$ membrane and 50-nm diameter reference pinholes were added [cf. Fig. 1(a)].

A commercial-grade simulator based on the finitedifference time-domain method [21] was used to calculate the electric field distribution in the object hole after excitation with $\lambda=780$-nm pump light pulses. The angle of incidence was $45^{\circ}$, and the electric field polarization was aligned parallel to the plane of incidence (i.e., along the long axis of the ellipse; cf. Fig. 1). The curved shape of the gold edge of the elliptical object hole reflects and focuses the light to form a standing wave with a very pronounced electric field enhancement. Because of the noncollinear geometry and the large dimension and gap size of the gold nanoislands, the field enhancement at the edge of these structures is negligible in comparison. Figure 1(b) displays the calculated distribution of the squared electric field $|E|^{2}$ with its first maximum having a lateral extension of $500 \mathrm{~nm}$ (FWHM) and being displaced from the center of the ellipse by $800 \mathrm{~nm}$. The electric field enhancement is normalized to the incoming light intensity and reaches a maximum of approximately $|E|^{2}=7$ at the surface of the magnetic

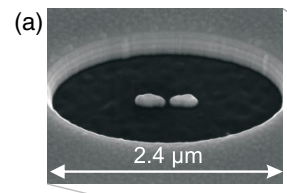

(b) electric field enhancement $|E|^{2}$

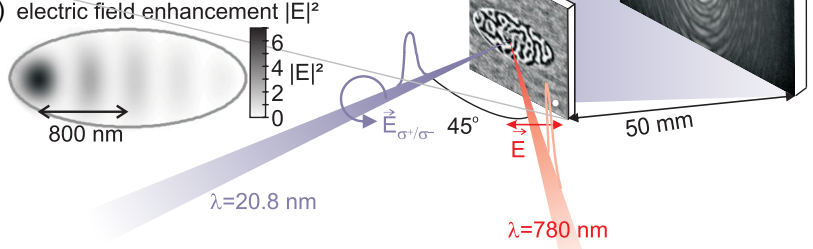

FIG. 1 (color online). Experimental setup. The black and white areas of the sample represent the in- and out-of-plane magnetized domains. (a) Scanning electron microscope image of the elliptical object hole with two gold nanoislands. (b) Calculated squared electric field $|E|^{2}$ after excitation with $p$-polarized, $\lambda=780-\mathrm{nm}$ light, incident under $45^{\circ}$.

multilayer, equivalent to a maximal excitation density of $9 \mathrm{~mJ} \mathrm{~cm}^{-2}$.

The magnetization holograms calculated by the difference of positive and negative helicity were transformed to in-plane Fourier coordinates [22] and reconstructed by a two-dimensional Fourier transform. The upper row of Fig. 2 shows the reconstructed domain patterns for an unpumped and pumped sample for selected delay times between optical excitation and holographic probing. The black and white areas within the elliptical field of view represent domains with magnetization $\vec{M}$ aligned either parallel or antiparallel to the incident x-ray beam direction. The nontransparent gold islands are masked. The magnetic domains of a width of $\approx 70 \mathrm{~nm}$ are clearly resolved. The $\mathrm{XMCD}$ contrast shows a significantly reduced magnetic contrast between oppositely magnetized domains in the upper part of the elliptical object hole, indicating a pronounced demagnetization by the locally enhanced optical excitation. A random measurement sequence of the different time delays ensures that nonreversible changes of the magnetic state do not introduce systematic timedependent trends; the unpumped image in Fig. 2(a) was measured after the time series. A comparison of the domain pattern in these images reveals some subtle permanent changes in the magnetic domain pattern, which we attribute to random intensity spikes of the FEL beam [19,23]. Careful inspection of the real-space images of the domain pattern reveals that the XMCD contrast shows a slight recovery for later times. The reappearance of magnetic domains clearly demonstrates that the loss of magnetic contrast due to the optical excitation does not originate from a time average of varying domain patterns in the repetitive, multishot experiment, but indeed from a reduction of contrast from a specific pattern. The lower row of Figs. 2(e)-(2h) shows the calculated moving variance of the XMCD contrast for each time step within $72 \times 72-\mathrm{nm}^{2}$ sub-blocks, chosen to be on the order of the domain size, 


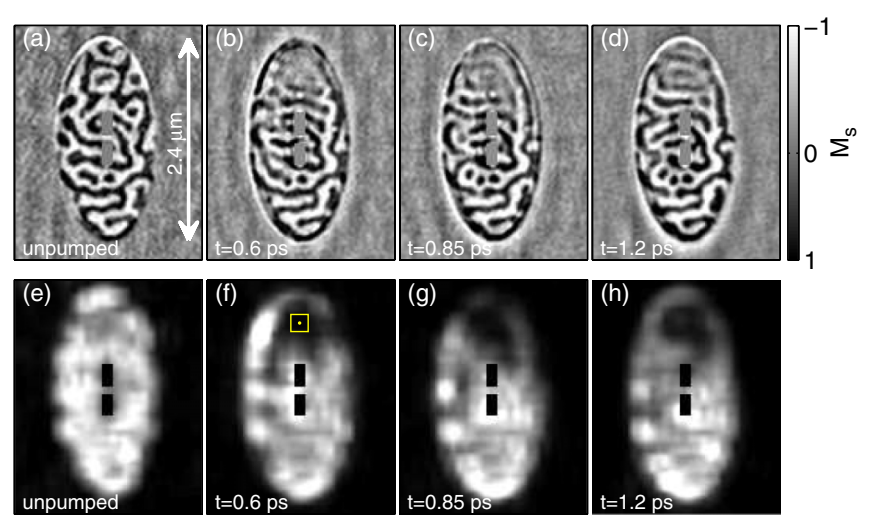

FIG. 2 (color online). Reconstructions of the magnetic domains for unpumped (a) and pumped samples for selected time delays (b)-(d). In the upper area of the elliptical object hole, the magnetic contrast is reduced. (e)-(h) Calculated variance of $72 \times 72-\mathrm{nm}^{2}$ areas of the magnetic contrast. For normalization, see text.

normalized, and scaled between $1 \%$ and $99 \%$. Since the variance is directly proportional to the magnetic domain contrast, or magnetization, the well-defined minimum in the upper part of the object hole corresponds to the localized loss of magnetization. Its position shows very good agreement with the first maximum of the calculated electric field enhancement. We do not observe an appreciable demagnetization due to the second maximum of the standing wave because of its significantly lower excitation density and its partial overlap with the upper gold nanoisland.

In Fig. 3(a) we plot the temporal evolution of the variance of a $200 \times 200-\mathrm{nm}^{2}$ area centered at the spatial position of maximal demagnetization [square rectangle in Fig. 2(f)] and normalized to the summed variance of the entire image. One observes an ultrafast drop in the variance, reaching a minimum after approximately $350 \mathrm{fs}$ and, subsequently, a $20 \%$ increase on a longer time scale. Therefore, one identifies the temporal behavior of a typical ultrafast demagnetization curve: an initial ultrafast loss of magnetization on a time scale of a couple of hundred femtoseconds, followed by a recovery of the magnetization within several picoseconds. This important observation together with a comparison of time-resolved magnetooptical Kerr effect (MOKE) measurements of Co-based multilayer [dash-dotted line in Fig. 3(a); also cf. Fig. 4(a)] allows us to establish the onset of demagnetization with an accuracy of better than $100 \mathrm{fs}$.

Figures 3(b), 3(c) summarize how the spatially localized demagnetization changes laterally as a function of time. Radial line scans centered at the minimum of the variance matrix are calculated within an angle range of $160^{\circ}$ and a radius of $r=600 \mathrm{~nm}$ [cf. the indicated area in the inset of Fig. 3(b)]. This ensures that the topography of the object hole itself is not superimposed with the magnetic contrast and that we exclusively analyze the region excited by the first maximum of the standing wave. The resulting profiles
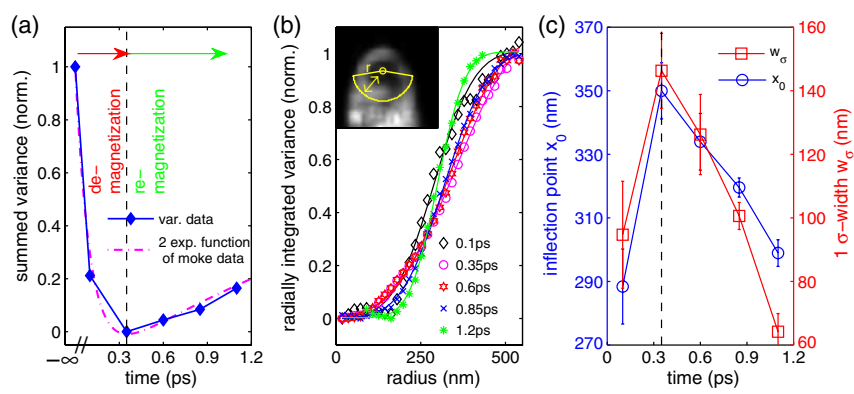

FIG. 3 (color online). (a) Normalized integrated variance as a function of time (line is a guide to the eye). Dotted line: double exponential function describing time-resolved MOKE measurements with $\tau_{\mathrm{RE}}=2.6 \mathrm{ps}$. The demagnetization time constant has been set to 100 fs. (b) Normalized radial integrated variance as a function of radius for different time delays with corresponding error functions, indicating the size of the demagnetized area. Inset: variance matrix showing the area of the radial integration. (c) Inflection point, $x_{0}$, and width, $\mathrm{w}_{\sigma}$, of the radial integrated variance as a function of time delay.

are then fitted with an error function [Fig. 3(b)], and their inflection points, $x_{0}$, and $1 \sigma$ widths, $w_{\sigma}$, are plotted as functions of time in Fig. 3(c). The error bars represent the reproducibility after changes in the center position $( \pm 50 \mathrm{~nm})$. One observes a rapid lateral increase of the demagnetized area, with the propagation front moving with a speed on the order of $0.2 \mathrm{~nm} \mathrm{fs}^{-1}$. For later times, the position of $x_{0}$ decreases again by $\approx 50 \mathrm{~nm}$ on a longer time scale. The width, $w_{\sigma}$, of the boundary between magnetized and demagnetized regions shows a similar behavior: an initial broadening of $\approx 40 \mathrm{~nm}(1 \sigma)$ within a couple of hundred femtoseconds is followed by its reduction $(\approx 80 \mathrm{~nm})$ for later times. A comparison between Figs. 3(a) and 3(c) shows that the temporal evolution of the lateral shape of the demagnetized area coincides with the two identified time ranges: the initial ultrafast demagnetization followed by the slower remagnetization.

We propose that the temporal evolution of the shift and width of the boundary between the demagnetized and magnetized regions for later times ( $t>350 \mathrm{fs})$ is a direct consequence of the fluence-dependent remagnetization times. A slowing down of the remagnetization process for high fluence has been shown experimentally and has been predicted by atomistic Landau-Lifshitz-Bloch and LandauLifshitz-Gilbert approaches [24-26] as well as by the microscopic three-temperature model of Koopmans et al. [10]. We have performed independent time-resolved magneto-optical Kerr experiments to extract the remagnetization times of comparable Co-based multilayers $\left[\mathrm{Si}_{3} \mathrm{~N}_{4}-\mathrm{Cr}(20) / \mathrm{Pt}(30) /\right.$ $\left.[\mathrm{Co}(4) / \mathrm{Pt}(7)]_{8} / \mathrm{Pt}(13)(\AA)\right]$. The measured transient magnetization curves are fitted by multiple exponential functions to yield remagnetization times as shown in Fig. 4(a). Assuming a maximal excitation density of approximately $9 \mathrm{~mJ} \mathrm{~cm}^{-2}$, we determine by linear extrapolation of our data a remagnetization time of $\tau_{\mathrm{RE}}=2.6 \mathrm{ps}$. An exponential function with a demagnetization (DE) constant of $\tau_{\mathrm{DE}}=0.1 \mathrm{ps}$ 

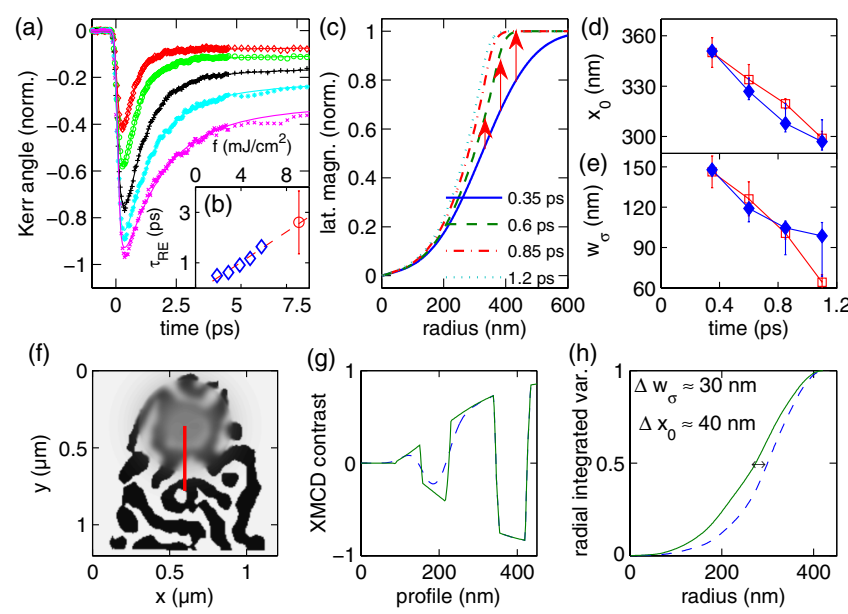

FIG. 4 (color online). (a) Measured time-resolved MOKE for fluences of 1.9 (diamonds), 2.9 (circles), 3.9 (plus signs), 4.8 (stars), and $5.8 \mathrm{~mJ} \mathrm{~cm}^{-2}$ (crosses). (b) Remagnetization times as a function of fluence; (red dot) extrapolation for an excitation of $9 \mathrm{~mJ} \mathrm{~cm}^{-2}$. (c) Calculated lateral magnetization assuming weighted remagnetization times $\tau_{\mathrm{RE}}$ between 0 and 2.6 ps for nondemagnetized and maximal demagnetized regions, respectively. (d) Inflection point, $x_{0}$, and (e) $1 \sigma$ width, $w_{\sigma}$, of the magnetization profile extracted from (c) and corresponding data from Fig. 3(c) (squares). (f) Real-space domain pattern [cf. Fig. 2(a)] with simulated local reduction of the XMCD contrast and softening of the domain walls. The red line indicates the position of profile shown in $(\mathrm{g})$ with (dashed line) and without (solid line) domain wall broadening. (h) Corresponding radial integrated variance with (dashed line) and without (solid line) domain wall broadening.

and the corresponding $\tau_{\mathrm{RE}}=2.6 \mathrm{ps}$ is plotted in Fig. 3(a) and shows good agreement with the experimental variance data. The fluence-dependent magnetization recovery will change the shape of the boundary, as strongly demagnetized regions will recover more slowly, whereas the weakly demagnetized part of the boundary will recover more quickly. We use the measured radial integrated profile for $350 \mathrm{fs}$ as shown in Fig. 3(b) as a starting point and calculate the time evolution using linearly weighted remagnetization times $\tau_{\mathrm{RE}}$ varying between 0 and $(2.5 \pm 1.5)$ ps for nonmagnetized and maximal demagnetized regions, respectively. The results are summarized in Figs. 4(c)-4(e): (c) shows how the shape of the boundary between magnetized and demagnetized regions changes as a function of time; the red arrows illustrate the nonlinear evolution. The corresponding shifts of the inflection point, $x_{0}$, and the width, $w_{\sigma}$, as a function of time are shown in (d) and (e). The error bars result from the estimated uncertainty in the maximal $\tau_{\mathrm{RE}}$. We not only observe that the fluence-dependent remagnetization time is able to explain the trend of the time-dependent shift and width of the magnetization profile but that the magnitude and time scale of these parameters are also well reproduced.

The initial ultrafast and pronounced shift of $x_{0}$ and increase in $w_{\sigma}$ of the magnetization profile suggest that subpicosecond transport processes of (spin-polarized) electrons, as recently discussed in theoretical and experimental work $[4,5,7-9,11]$, may play a decisive role. Laserexcited nonequilibrium spin-polarized electrons show anomalous transport properties, characterized by a transition from a ballistic to a diffusive regime, which due to distinct energy-dependent velocities and lifetimes of majority and minority spins leads to ultrafast spin dynamics [11]. The energy-dependent velocities of the electrons are on the order of $v=0.5 \mathrm{~nm} \mathrm{fs}^{-1}$ [27]; hence, for early times $t$, when electron transport is dominated by ballistic motion $(x=v t)$, spin relocation $x$ can reach several tens of nanometers. Because of the magnetic inhomogeneity of our sample, consisting of a nanoscale network of magnetic domains, majority spins with higher mobility will rapidly accumulate at the boundary walls of the neighboring domains where they become minority carriers and are trapped. Note that this implies that the microscopic flow of the spin-polarized electrons will depend on the specific configuration of the magnetic domain network and will lead to a softening of the domain wall boundaries. This effect has recently been observed by resonant x-ray scattering of magnetically inhomogeneous $\mathrm{Co} / \mathrm{Pt}$ samples and corroborated by Monte Carlo simulations. After 300-fs superdiffusive spin transport was estimated to broaden the domain wall width by approximately $30 \mathrm{~nm}$ [8]. In the following, we show that this scenario is consistent with our experimental data: Fig. 4(f) shows the digitized real-space domain pattern $\left(\mathrm{M}_{s}=1\right.$ or -1 ) of Fig. 2(a) with a calculated locally reduced XMCD contrast according to the first maximum of the electric field enhancement shown in Fig. 1(b). To approximate the effect of spin-polarized currents, we smooth the domain wall width by up to $30 \mathrm{~nm}$ weighted by the excitation density; a cross section along the red line with and without softening of the domain profiles is shown in Fig. $4(\mathrm{~g})$. We then repeat the same analysis carried out with the experimental data set; i.e., we calculate the corresponding variance matrix (not shown) and plot its radial integration in Fig. 4(h). Here, we observe a shift and a broadening of the magnetization profile by $\Delta x_{0} \approx$ $40 \mathrm{~nm}$ and $\Delta w_{\sigma} \approx 30 \mathrm{~nm}$, respectively, on the same order as the measured values between 100 and 350 fs shown in Fig. 3(c). We like to point out that a scenario in which impurity or phonon spin-flip scattering events [10] after an ultrafast (ballistic) transport of nonequilibrium electrons would also lead to an ultrafast spatial rearrangement of magnetization. With the anomalous spin-dependent transport properties of excited electrons, this process is expected to lead to similar changes of the lateral magnetization profile. A distinction and quantification of these possibly competing processes is challenging and calls for further experimental efforts.

In conclusion, we have shown that an ultrafast optical demagnetization stimulus can be spatially localized via a tailored microresonator. We observe the spatial extent of the resulting demagnetization in a magnetic domain network in real space via time-resolved FTH with 100-fs temporal and sub-100-nm spatial resolution. Our 
experimental observation suggests that intrinsic nonlocal mechanisms lead to an ultrafast change of the spatial magnetization profile, supporting the notion that ultrafast transport of spin-polarized electrons plays an important role in magnetic inhomogeneous materials. This implies that engineered magnetic domain sizes will allow controlling the time scale and the spatial confinement of the optically manipulated magnetic order. Furthermore, using optical antennas, wedge-shaped metallic plates, for example, for plasmonic driven light enhancement will allow nanoscale spatial control of the magnetic order, which is particularly intriguing for localized all-optical switching applications in nanoscale devices.

We are indebted to Maya Kiskinova for support during the FEL experiment and discussion of the manuscript. We thank Guillaume Beutier for help during the experiment. C. v. K. S., B. P., C. M. G., and S. E. gratefully acknowledge support from the German Bundesministerium für Bildung und Forschung under Contract No. 05K10KTB/FSP-301 ("MPScatt"). The work of F. C., E. P., and N. M. was funded by the FERMI project of Elettra-Sincrotrone Trieste, partially supported by the Ministry of University and Research under Grants No. FIRB-RBAP045JF2 and No. FIRBRBAP06AWK3. L. M. thankfully acknowledges financial support from DFG within SFB 925. J. B., B. V., and J. L. acknowledge support from the CNRS through the PEPS SASELEX and from the French ANR via the FEMTO-XMAG project. Additionally, B. V. acknowledges financial support from the LabEx PALM through the RAMSES project.

*c.vonkorffschmising@tu-berlin.de

†Present address: Division of Synchrotron Radiation Research, Department of Physics, Lund University, S-22100 Lund, Sweden

[1] C. D. Stanciu, F. Hansteen, A. V. Kimel, A. Kirilyuk, A. Tsukamoto, A. Itoh, and T. Rasing, Phys. Rev. Lett. 99, 047601 (2007).

[2] K. Vahaplar, A. M. Kalashnikova, A. V. Kimel, D. Hinzke, U. Nowak, R. Chantrell, A. Tsukamoto, A. Itoh, A. Kirilyuk, and T. Rasing, Phys. Rev. Lett. 103, 117201 (2009).

[3] A. Kirilyuk, A. V. Kimel, and T. Rasing, Rep. Prog. Phys. 76, 026501 (2013).

[4] G. Malinowski, F. Dalla Longa, J. H. H. Rietjens, P. V. Paluskar, R. Huijink, H. J. M. Swagten, and B. Koopmans, Nat. Phys. 4, 855 (2008).

[5] D. Rudolf, C. L.-O. Vorakiat, M. Battiato, R. Adam, J. M. Shaw, E. Turgut, P. Maldonado, S. Mathias, P. Grychtol, H. T. Nembach, T. J. Silva, M. Aeschlimann, H. C. Kapteyn, M. M. Murnane, C. M. Schneider, and P. M. Oppeneer, Nat. Commun. 3, 1037 (2012).

[6] T. Kampfrath, M. Battiato, P. Maldonado, G. Eilers, J. Noetzold, S. Maehrlein, V. Zbarsky, F. Freimuth,
Y. Mokrousov, S. Bluegel, M. Wolf, I. Radu, P. M. Oppeneer, and M. Muenzenberg, Nat. Nanotechnol. 8, 256 (2013).

[7] E. Turgut, C. L. Vorakiat, J. M. Shaw, P. Grychtol, H. T. Nembach, D. Rudolf, R. Adam, M. Aeschlimann, C. M. Schneider, T. J. Silva, M. M. Murnane, H. C. Kapteyn, and S. Mathias, Phys. Rev. Lett. 110, 197201 (2013).

[8] B. Pfau et al., Nat. Commun. 3, 1100 (2012).

[9] B. Vodungbo, J. Gautier, G. Lambert, A. B. Sardinha, M. Lozano, S. Sebban, M. Ducousso, W. Boutu, K. Li, B. Tudu, M. Tortarolo, R. Hawaldar, R. Delaunay, V. Lopez-Flores, J. Arabski, C. Boeglin, H. Merdji, P. Zeitoun, and J. Luening, Nat. Commun. 3, 999 (2012).

[10] B. Koopmans, G. Malinowski, F. Dalla Longa, D. Steiauf, M. Faehnle, T. Roth, M. Cinchetti, and M. Aeschlimann, Nat. Mater. 9, 259 (2010).

[11] M. Battiato, K. Carva, and P. M. Oppeneer, Phys. Rev. Lett. 105, 027203 (2010).

[12] M. H. Kryder, E. C. Gage, T. W. Mcdaniel, W. A. Challener, R. E. Rottmayer, G. Ju, Y.-T. Hsia, and M. F. Erden, Proc. IEEE 96, 1810 (2008).

[13] E. Betzig, J. K. Trautman, R. Wolfe, E. M. Gyorgy, P. L. Finn, M. H. Kryder, and C. H. Chang, Appl. Phys. Lett. 61, 142 (1992).

[14] T. Matsumoto, T. Shimano, H. Saga, H. Sukeda, and M. Kiguchi, J. Appl. Phys. 95, 3901 (2004).

[15] T. Matsumoto, Y. Anzai, T. Shintani, K. Nakamura, and T. Nishida, Opt. Lett. 31, 259 (2006).

[16] W. A. Challener, C. Peng, A. V. Itagi, D. Karns, W. Peng, Y. Peng, X.-M. Yang, X. Zhu, N. J. Gokemeijer, Y. T. Hsia, G. Ju, R. E. Rottmayer, M. A. Seigler, and E. C. Gage, Nat. Photonics 3, 303 (2009).

[17] S. Eisebitt, J. Luning, W. F. Schlotter, M. Lorgen, O. Hellwig, W. Eberhardt, and J. Stohr, Nature (London) 432, 885 (2004).

[18] F. Capotondi et al., Rev. Sci. Instrum. 84, 051301 (2013)

[19] C. Gutt, S. Streit-Nierobisch, L.-M. Stadler, B. Pfau, C. M. Günther, R. Könnecke, R. Frömter, A. Kobs, D. Stickler, H. P. Oepen, R. R. Fäustlin, R. Treusch, J. Feldhaus, E. Weckert, I. A. Vartanyants, M. Grunze, A. Rosenhahn, T. Wilhein, S. Eisebitt, and G. Grübel, Phys. Rev. B 81, 100401 (2010).

[20] C. Gahl, A. Azima, M. Beye, M. Deppe, K. Doebrich, U. Hasslinger, F. Hennies, A. Melnikov, M. Nagasono, A. Pietzsch, M. Wolf, W. Wurth, and A. Foehlisch, Nat. Photonics 2, 165 (2008).

[21] Lumerical Solutions, Inc., Vancouver, BC V6E 3L2, Canada.

[22] S. Schaffert, B. Pfau, J. Geilhufe, C. M. Günther, M. Schneider, C. von Korff Schmising, and S. Eisebitt, New J. Phys. 15, 093042 (2013).

[23] T. Wang et al., Phys. Rev. Lett. 108, 267403 (2012).

[24] U. Atxitia, O. Chubykalo-Fesenko, J. Walowski, A. Mann, and M. Münzenberg, Phys. Rev. B 81, 174401 (2010).

[25] N. Kazantseva, U. Nowak, R. W. Chantrell, J. Hohlfeld, and A. Rebei, Europhys. Lett. 81, 27004 (2008).

[26] R. Chimata, A. Bergman, L. Bergqvist, B. Sanyal, and O. Eriksson, Phys. Rev. Lett. 109, 157201 (2012).

[27] V. P. Zhukov, E. V. Chulkov, and P. M. Echenique, Phys. Rev. B 73, 125105 (2006). 